\title{
Avaliação neurológica pelo método Dubowitz em recém-nascidos prematuros com idade corrigida de termo comparada a de nascidos a termo
}

\author{
Neonatal neurological assessment by the Dubowitz method in preterm infants at term corrected age \\ compared with term infants
}

Marina Ortega Golin ${ }^{1}$, Fabíola Isabel S. de Souza ${ }^{2}$, Roseli Oselka S. Sarni

\section{RESUMO}

Objetivo: Comparar a avaliação neurológica de recém-nascidos pré-termo em idade de termo com a de recém-nascidos a termo e verificar a presença de associação entre alterações na avaliação neurológica e características e intercorrências clínicas nos prematuros.

Método: Estudo transversal de 60 nascidos a termo e 30 prematuros do Hospital Estadual Mário Covas, em Santo André (SP), avaliados pelo método Dubowitz. Os pontos de corte da pontuação total foram 30,5 e 26 para comparar recém-nascidos pré-termo e a termo, respectivamente. Os prematuros foram avaliados com idade correspondente ao termo, na $37^{\mathrm{a}}$ semana, e os do grupo controle, 48 horas após o nascimento. Todos os neonatos foram avaliados pela mesma examinadora, entre as mamadas, nos estágios 4 e 5 de sono e vigília. Para análise estatística, utilizaram-se testes de associação.

Resultados: 90\% dos nascidos pré-termo não atingiram a pontuação esperada para a idade de termo $(p<0,0001)$, com alterações mais evidentes nas dimensões: tônus, tipo de tônus e movimentos. Dentre os prematuros, $57 \%$ não atingiram pontuação total igual ou superior a 26. A sepse neonatal foi a única intercorrência associada de maneira significante, com pontuações abaixo da normalidade em recém-nascidos pré-termo $(p=0,009)$.

Conclusão: Os recém-nascidos pré-termo avaliados com 37 semanas de idade corrigida, quando comparados aos nascidos a termo, apresentaram menor pontuação no exame neurológico e apenas a sepse se associou a alterações neurológicas.

Palavras-chave: exame neurológico; recém-nascido; prematuro; deficiências do desenvolvimento.

\section{ABSTRACT}

Objective: To compare neonatal neurological assessment of preterm newborn infants at term corrected age with term infants, and to identify the presence of association between neurologic abnormalities in preterm infants and demographic characteristics and neonatal clinical conditions.

Methods: This cross-sectional study enrolled 60 term and 30 preterm infants born at the Mario Covas State Hospital in Santo André, São Paulo, Brazil. The Dubowitz method was applied to assess neurobehavior. The total score cut-offs of 30.5 and 26 were used to compare respectively preterm and term infants and to analyze the variables related to performance in the preterm group. Preterm newborns were evaluated at 37 post-conceptual weeks and the control group was evaluated 48 hours after birth. All newborns were evaluated by the same neurologist, between feedings and at sleep-awake stages 4 or 5 . Statistical analysis was performed by association tests.

Results: $90 \%$ of the preterm infants did not reach the expected score for term age. Underachievement was seen mainly in the domains of muscle tonus, type of muscle tonus and movements. Among the 30 preterm infants included in
Instituição: Faculdade de Medicina do ABC (FMABC), Santo André, SP, Brasil

${ }^{1}$ Fisioterapeuta; Mestre em Ciências da Saúde pela FMABC; docente dos cursos de Fisioterapia da Universidade Nove de Julho (Uninove) e FMABC, Santo André, SP, Brasil

${ }^{2}$ Mestre em Ciências pela Universidade Federal de São Paulo (Unifesp); Médica do Serviço de Nutrologia do Departamento de Pediatria da FMABC, Santo André, SP, Brasil

${ }^{3}$ Doutora em Medicina pela Unifesp; médica assistente do Departamento de Pediatria da Unifesp e Professora assistente do Departamento de Pediatria da FMABC, Santo André, SP, Brasil
Endereço para correspondência

Marina Ortega Golin

Praça Universo, 96, apto 25B - Vila Formosa

CEP 03362-020 - São Paulo/SP

E-mail: marinaog@uninove.br

Recebido em: 12/10/08

Aprovado em: 31/5/09 
the study, $57 \%$ did not reach a total score $\geq 26$. Sepsis was the only complication significantly associated with neurological impairment ( $p=0.009)$.

Conclusion: Preterm newborn infants at 37 weeks corrected age do not accomplish the neurologic performance of term infants. Sepsis was the only clinical variable associated with neurological disabilities.

Key-words: neurologic examination; infant, newborn; infant, premature; developmental disabilities.

\section{Introdução}

Os avanços científicos e tecnológicos nos cuidados neonatais possibilitaram redução significativa na mortalidade de recém-nascidos pré-termo (RNPT); entretanto, há evidências de que o aumento na sobrevida foi acompanhado por maior ocorrência de disfunções neurológicas ${ }^{(1,2)}$, sendo encontradas incidências elevadas de paralisia cerebral (10\%), déficits sensoriais $(3,5 \%)$ e cognitivos $(24 \%)^{(3)}$.

Em prematuros, a identificação e o tratamento precoce dos desvios do desenvolvimento são estratégias efetivas para minimizar as consequências da lesão encefálica e promover melhor qualidade de vida a longo prazo ${ }^{(4)}$. A intervenção multidisciplinar precoce é essencial devido à grande plasticidade cerebral do lactente. Os primeiros oito meses de vida constituem o período de desenvolvimento mais rápido e intenso do sistema nervoso, o que implica maior capacidade de reorganização frente a uma agressão(s).

Nas últimas duas décadas, o advento de técnicas mais avançadas de exames subsidiários não reduziu o valor da avaliação clínica dos recém-nascidos ( $\mathrm{RN})$. Na verdade, o uso associado de ambos os recursos possibilitou o aperfeiçoamento de valores de predição de lesões neurológicas, uma vez que a avaliação clínica continua sendo o recurso mais acessível à maioria dos hospitais ${ }^{(6)}$.

Há vários métodos de avaliação neurológica neonatal. $\mathrm{O}$ proposto por Dubowitz e Dubowitz é reconhecido internacionalmente e largamente utilizado em pesquisas por ser aplicável tanto em RNPT quanto em nascidos a termo $(\mathrm{RNT})^{(7)}$. Além de sua validade científica, esse método possui várias vantagens: é de fácil aplicabilidade, possui instruções para realização de cada item, podendo ser utilizado por qualquer profissional da saúde com conhecimento na área de neurologia ${ }^{(8,9)}$.

Os resultados de inúmeras pesquisas na literatura internacional validam o uso desse método para identificar pacientes expostos a lesões neurológicas no período neonatal. Alguns estudos transversais avaliaram tanto RNPT como $\mathrm{RNT}^{(10)}$, ou apenas $\mathrm{RNPT}^{(7)}$, observando associação significante entre a avaliação clínica e as alterações demonstradas por métodos de imagem, como a presença de hemorragias peri e intraventriculares no ultrassom transfontanelar e leucomalácia periventricular ou encefalopatia hipóxico-isquêmica na ressonância magnética. Além disso, estudos coorte encontraram associação entre alterações na avaliação neonatal e a presença de paralisia cerebral no primeiro ano de vida ${ }^{(11,12)}$.

No Brasil, há apenas dois trabalhos publicados ${ }^{(4,13)}$ que descreveram o comportamento neurológico de RNPT segundo esse método. Fato que reflete a escassez de informações sobre as respostas esperadas em nossa população, o que provavelmente limita sua aplicação na rotina clínica das unidades neonatais para identificar pacientes com prováveis disfunções do desenvolvimento.

O objetivo do presente estudo foi comparar a avaliação neurológica neonatal de RNPT na idade corrigida de termo com a de RNT. Além disso, buscou-se, em RNPT, relacionar as alterações da avaliação neurológica com as características e as intercorrências clínicas nos períodos pré, peri e neonatal.

\section{Métodos}

Trata-se de um estudo transversal com $90 \mathrm{RN}$, sendo 30 RNPT (grupo estudo) e 60 RNT (grupo controle). As avaliações neurológicas neonatais foram realizadas no Hospital Estadual Mário Covas de Santo André (SP), ligado à Faculdade de Medicina do ABC (FMABC), que é um centro de referência para o encaminhamento de gestantes de alto risco da região. O projeto foi aprovado pelo Comitê de Ética em Pesquisa da FMABC.

A amostra de pacientes foi obtida de maneira consecutiva até atingir o número de $\mathrm{RN}$ estimado pelo cálculo amostral, tanto no grupo estudo quanto no controle, resultando em período de coleta de dez meses. Para o cálculo da amostra, foi considerada a prevalência relatada na literatura de alterações no exame neurológico em ambas as populações, sendo $50 \%$ em RNPT e $10 \%$ em RNT de baixo risco. Estimou-se $\alpha=0,05$ (bidirecional) e $\beta=0,20$; chegando-se a $24 \mathrm{RN}$ por grupo. Acrescentou-se $20 \%$ de estimativa de perda, totalizando 30 RN por grupo, na proporção 1 para $2^{(14)}$. Não foram estabelecidos critérios de pareamento para casos e controles.

O critério de inclusão para o grupo estudo foi idade gestacional ao nascimento até 36 semanas. Os critérios de exclusão para o grupo estudo foram: necessidade de ventilação mecânica no momento da avaliação; presença de malformações 
congênitas e síndromes neurológicas ou presença de desvios fenotípicos; evidências de infecções congênitas, doenças metabólicas, endócrinas e gemelaridade. Já para o grupo controle foram considerados para a inclusão: idade gestacional ao nascimento entre 37 e 41 semanas; peso superior ou igual a 2500g; ausência de intercorrências no parto e no período neonatal e Apgar no quinto minuto maior ou igual a sete. Nos dois grupos, para inclusão, foi lido e assinado pelas mães o Termo de Consentimento Livre e Esclarecido.

Os RN foram classificados de acordo com o peso ao nascimento e o esperado em pequenos (peso inferior ao percentil 10 para a idade gestacional), adequados (peso entre os percentis 10 e 90) ou grandes para a idade gestacional (acima do percentil 90) ${ }^{(15)}$. A idade gestacional foi estimada preferencialmente pela data da última menstruação ou pelo resultado do ultrassom do primeiro trimestre de gestação e, em último caso, pela avaliação clínica pelo método de Capurro $^{(16)}$, que faz parte da rotina dos neonatologistas da unidade hospitalar na qual foi realizada a pesquisa.

A partir de questionário pré-codificado foram coletados: 1) Dados sobre as características maternas e da gestação: realização de pré-natal, peso e altura pré-gestacional (cálculo do índice de massa corpórea); ganho ponderal; condição socioeconômica (inferior ou superior a meio salário mínimo); nível de escolaridade (inferior ou superior a quatro anos); uso de tabaco (sim/não), álcool (sim/não) e drogas ilícitas (sim/não) durante a gestação; uso de ferro e antibióticos; hipertensão arterial; diabetes e outras doenças; presença de sangramento (dado referido); rotura prematura de membranas e tipo de parto. 2) Características do RN: sexo, idade gestacional e peso ao nascimento; Apgar no primeiro e quinto minutos de vida; complicações respiratórias e cardíacas; icterícia; hipoglicemia; infecções neonatais; alterações no ultrassom transfontanelar; utilização e duração de antibioticoterapia; uso e permanência em ventilação mecânica e oxigenoterapia.
As avaliações foram realizadas sempre pelo mesmo examinador, não sendo realizado treinamento para habilitação. Os RNPT foram avaliados com idade correspondente ao termo, durante a $37^{\mathrm{a}}$ semana e, os do grupo controle, 48 horas após o nascimento. Todos os $\mathrm{RN}$ foram avaliados entre as mamadas, nos estágios 4 e 5 de sono e vigília, conforme preconizado por Brazelton $^{(17)}$. O protocolo de avaliação utilizado foi o proposto por Dubowitz ${ }^{(8)}$, que consiste de 34 itens agrupados em seis dimensões: tônus (dez itens), tipo de tônus (cinco itens), reflexos (seis itens), movimentos (três itens), sinais anormais (três itens) e comportamento (sete itens). Há um diagrama com as pontuações e cada item apresenta de três a cinco opções de respostas esperadas. A composição das pontuações é calculada pela soma de todos os itens dentro de cada dimensão. De acordo com a resposta obtida em cada item - normal, intermediária ou anormal - atribuem-se as pontuações 1; 0,5 e 0; respectivamente. A faixa de normalidade da pontuação total varia de 30,5 a 34. A pontuação inferior a 30,5 foi adotada para comparar os grupos (RNPT e RNT). Para a análise dos RNPT e das variáveis associadas a diferentes desempenhos, foi utilizada a pontuação de 26 , proposta pelos mesmos autores ${ }^{(18)}$.

Os dados foram expressos em tabelas de frequências. Para comparar as variáveis qualitativas e os itens da avaliação, foram utilizados o teste do qui-quadrado ou teste exato de Fisher e a Odds Ratio. Foi considerado um nível de significância de 5\% para todos os testes.

\section{Resultados}

As características maternas estão mostradas na Tabela 1. A assistência pré-natal foi observada em $93 \%$ das mães dos RNPT e em 95\% das mães dos RNT. Apenas duas (3\%) mães do grupo RNT referiram uso de drogas ilícitas na gestação. Diabetes melito foi observado em apenas seis (20\%) gestantes no grupo de RNPT.

Tabela 1 - Caracterização das condições maternas gestacionais e morbidades associadas

\begin{tabular}{lrcc}
\hline & Termo $(\mathbf{n}=\mathbf{6 0})$ & Pré-termo $(\mathbf{n}=\mathbf{3 0})$ & Valor de $\boldsymbol{p}$ \\
\hline IMC $<18,5$ ou $>25 \mathrm{~kg} / \mathrm{m}^{\mathbf{2}}$ pré-gestacional & $3(5 \%)$ & $5(17 \%)$ & 0,037 \\
Uso de tabaco & $18(30 \%)$ & $9(30 \%)$ & 0,509 \\
Uso de álcool & $2(3 \%)$ & $1(3 \%)$ & 0,709 \\
Uso de vitamina na gestação & $42(70 \%)$ & $19(63 \%)$ & 0,342 \\
Uso de ferro na gestação & $39(56 \%)$ & $20(67 \%)$ & 0,534 \\
Uso de antibióticos na gestação & $12(20 \%)$ & $10(33 \%)$ & 0,130 \\
Hipertensão arterial sistêmica & $7(12 \%)$ & $10(33 \%)$ & 0,016 \\
Sangramento & $6(10 \%)$ & $11(37 \%)$ & 0,004 \\
Rotura prematura de membranas & $5(8 \%)$ & $7(23 \%)$ & 0,053 \\
\hline
\end{tabular}

IMC: índice de massa corpórea. 
Nos RNPT, a mediana do peso ao nascer foi $1655 \mathrm{~g}$ (variação: 705-3930) e da idade gestacional, 33 semanas (variação: 25-33). Apenas um $\mathrm{RN}$ apresentou Apgar de quinto minuto menor que 7. Quanto ao peso ao nascer, 17 (57\%) eram baixo peso, sete (23\%) muito baixo peso e três (10\%) extremo baixo peso. Quanto à classificação do peso ao nascer, em relação à idade gestacional, um (3\%) era pequeno e um (3\%) grande para a idade gestacional. Entre os RNPT, a icterícia esteve presente em 13 (43\%), a síndrome do desconforto respiratório em 22 (73\%), a cardiopatia congênita não complexa em 14 (47\%), a sepse em 25 (83\%), a necessidade de exsanguíneotransfusão em dois (7\%), a hipoglicemia em sete (23\%), o uso de oxigenoterapia em 29 (97\%) e de ventilação mecânica em 16 (53\%). Entre os RNPT que utilizaram antibióticos, ventilação mecânica e oxigenoterapia no período neonatal, a mediana do tempo de uso foi de 16 dias (variação: 6-65), 9 dias (variação: 2-90) e 5 dias (variação: 1-130), respectivamente.

A avaliação neurológica neonatal foi realizada nos RNT com dois dias de vida e, nos RNPT, com mediana de 22 dias de vida (variação: 3-97) e peso de $1945 \mathrm{~g}$ (variação: 12702715). Dos RNPT, 90\% não atingiram a pontuação esperada para a idade de termo, variando essa pontuação entre 5 e 30 . As dimensões tônus, tipo de tônus, reflexos, movimentos e sinais anormais mostraram diferença significante entre os nascidos a termo e os prematuros (Tabela 2).

A Tabela 3 descreve razões de chance $(\mathrm{OR})$ para alterações na avaliação neurológica de RNPT, adotando pontuação de corte de 26. Nessa população, observou-se que 17/30 (57\%) apresentaram pontuação inferior a 26 , havendo associação entre a sepse e esse desfecho $(p=0,009)$.

O Gráfico 1 mostra o percentual de RNPT com pontuações esperadas para a normalidade nas diversas dimensões e na avaliação global. Em relação às pontuações obtidas em cada item da avaliação em ambos os grupos, os RNPT apresentaram pontuação inferior em comparação aos RNT em 22 dos 34 itens avaliados: postura $(p=0,035)$, recolhimento dos braços $(p=0,012)$, tração dos braços $(p<0,001)$, recolhimento das pernas $(p=0,003)$, tração das pernas $(p=0,002)$, ângulo poplíteo $(p=0,005)$, controle da cabeça $1-$ extensor $(p<0,01)$, controle da cabeça $2-$ flexor $(p<0,001)$, intervalo da cabeça puxado para sentar $(p<0,001)$, suspensão ventral $(p<0,001)$, tônus extensor do pescoço $(p=0,012)$, reflexos $(p=0,011)$, $\operatorname{sucção~}(p<0,001)$, preensão plantar $(p<0,001)$, reflexo de Moro

Tabela 2 - Distribuição de recém-nascidos com pontuação abaixo da esperada

\begin{tabular}{lcccc}
\hline & Termo $(\mathbf{n = 6 0})$ & $\begin{array}{c}\text { Pré-termo } \\
(\mathbf{n = 3 0 )}\end{array}$ & OR (IC95\%) & Valor de $\boldsymbol{p}$ \\
\hline Pontuação total<30,5 & $8(13 \%)$ & $27(90 \%)$ & $58(12-319)$ & $<0,0001$ \\
Tônus $<10$ & $3(5 \%)$ & $24(80 \%)$ & $76(15-455)$ & $<0,0001$ \\
Tipo de tônus $<5$ & $39(65 \%)$ & $29(97 \%)$ & $16(2-329)$ & $<0,0001$ \\
Reflexos<4 & 0 & $11(37 \%)$ & Não definido & $<0,0001$ \\
Movimento<3 & $11(18 \%)$ & $27(90 \%)$ & $40(9-204)$ & $<0,0001$ \\
Sinais anormais<3 & $4(7 \%)$ & $9(30 \%)$ & $6(2-26)$ & 0,008 \\
Comportamento<6 & $20(33 \%)$ & $14(47 \%)$ & $1,8(1-5)$ & 0,253 \\
\hline
\end{tabular}

OR: Odds Ratio.

Tabela 3 - Razão de chance para alteração na pontuação total da avaliação neurológica neonatal de recém-nascidos pré-termo, segundo método Dubowitz

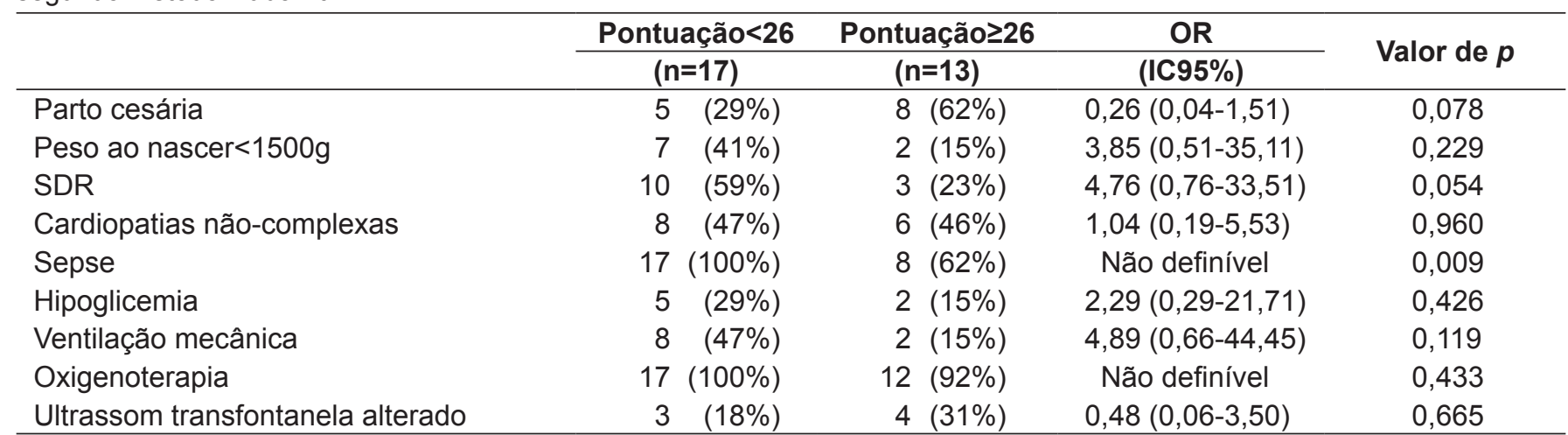

SDR: síndrome do desconforto respiratório; OR: Odds Ratio. 


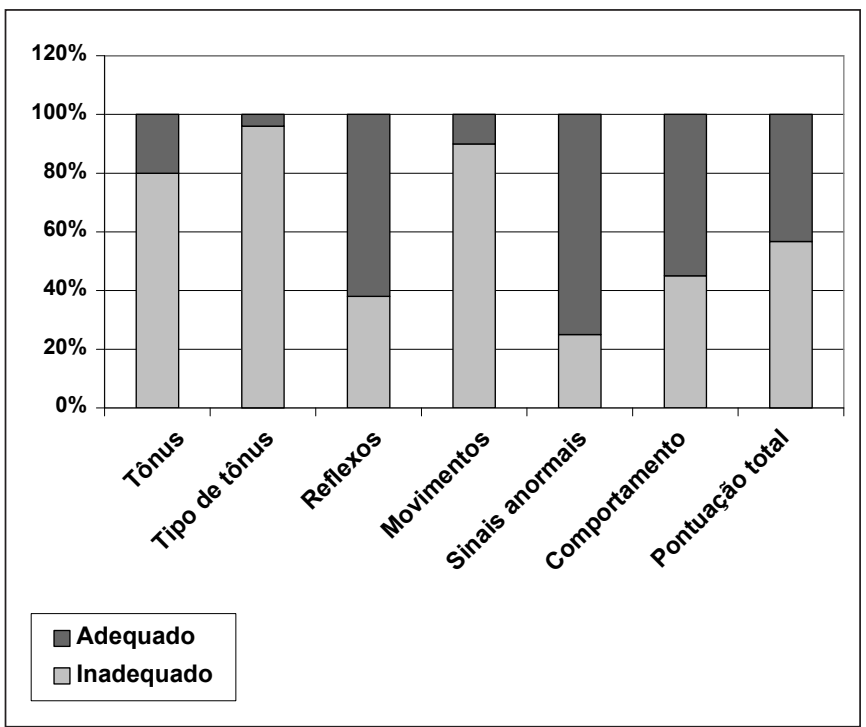

Gráfico 1 - Percentual de adequação das dimensões e da pontuação total da avaliação neurológica em recém-nascidos pré-termo.

( $p=0,034)$, movimentos espontâneos - quantidade $(p<0,001)$, movimentos espontâneos - qualidade $(p<0,001)$, tremor $(p=0,015)$, orientação visual $(p=0,002)$ e alerta $(p=0,002)$.

A diferença no desempenho nos diversos itens entre os RNPT com pontuação total igual ou superior a 26 daqueles com pontuação inferior a 26 está demonstrada no Gráfico 2.

\section{Discussão}

Neste estudo, 90\% dos RNPT não atingiram a pontuação esperada pelo método de Dubowitz, sendo a sepse neonatal o único fator de risco que se associou a essa baixa pontuação. A relação entre sepse e lesões neurológicas foi sugerida em um estudo de coorte prospectiva por O'Shea et al ${ }^{(19)}$, ao acompanharem 984 crianças de muito baixo peso ao longo do primeiro ano de vida. Os autores notaram associação entre a presença de sepse na primeira semana de vida e o desenvolvimento de paralisia cerebral.

As pesquisas atuais mostram que os fatores de risco envolvidos na gênese da lesão neurológica em RNPT não agem de maneira isolada, o que dificulta a análise da contribuição individual de cada fator. Nos RNPT, em contraste aos RNT, os fatores de risco neonatais desempenham papel de destaque na gênese de lesões neurológicas ${ }^{(20)}$, fato esperado devido à maior fragilidade do sistema nervoso central e ao grande número de complicações resultantes da própria imaturidade dos sistemas.

A infecção é apontada pela literatura atual como uma das causas primárias de lesão na substância branca encefálica de
RNPT, uma vez que torna as células do sistema nervoso central vulneráveis diante de uma agressão isquêmica até mesmo leve, que não seria suficiente para causar morte neuronal em outras circunstâncias ${ }^{(21)}$. Outros fatores de risco para lesões neurológicas são citados, destacando-se baixo peso e idade gestacional ao nascimento, anormalidades no ultrassom de crânio e desconforto respiratório ${ }^{(7,10-12,18,22,23)}$, entretanto, neste estudo não foram observadas tais relações. A idade correspondente ao termo é considerada a época adequada para a avaliação neurológica de RNPT. Nesse período, mesmo os RNPT extremos apresentam tônus e reflexos similares aos RNT e, assim, a avaliação é mais acurada ${ }^{(18)}$. No presente estudo, todos os RNPT foram avaliados na $37^{\mathrm{a}}$ semana, pois no hospital estudado muitos recebem alta logo após atingirem a idade de termo, se tiverem peso adequado.

Os estudos nacionais que empregaram o método Dubowitz utilizaram a pontuação de referência proposta pelos autores e analisaram o desempenho de RNT de baixo risco. Entretanto, outros fatores, como os ambientais, culturais, socioeconômicos e físicos, também podem interferir na avaliação de populações em diferentes países ${ }^{(24)}$. São escassos e com amostra pequena os estudos que tentaram identificar as características do método em populações diferentes. Um deles foi realizado em $58 \mathrm{RNT}$ vietnamitas sem evidências de intercorrências clínicas, nos quais se aplicou uma versão resumida do método Dubowitz. Em dez dos 25 itens avaliados, a pontuação foi significantemente menor. Com base nesses dados, os autores sugeriram que a pontuação de referência deveria ser usada com cuidado em trabalhos com diferentes populações em outros países, pois refletiria o esperado para um grupo populacional específico, podendo sofrer influência de fatores nutricionais e socioeconômicos regionais ${ }^{(25)}$.

De acordo com a pesquisa citada acima, no presente estudo optou-se por avaliar uma amostra de RNT local, sem evidências de intercorrências clínicas. Dos 60 RNT avaliados, $13 \%$ não obtiveram pontuações entre 30,5 e 34 . Tal achado difere dos resultados do estudo utilizado para compor a pontuação-referência do método, no qual apenas $5 \%$ não atingiram a pontuação acima ${ }^{(8)}$. Assim, é possível que a avaliação dos RNPT antes das 40 semanas completas de idade pós-concepcional e a realização do exame em condições diversas do estudo original possam ter contribuído para tais resultados.

A maior parte dos estudos que descreveram o comportamento neurológico de RNPT, segundo a versão atualizada do método, evidencia percentual significativo de pacientes com pontuação abaixo do esperado. São mencionadas proporções 


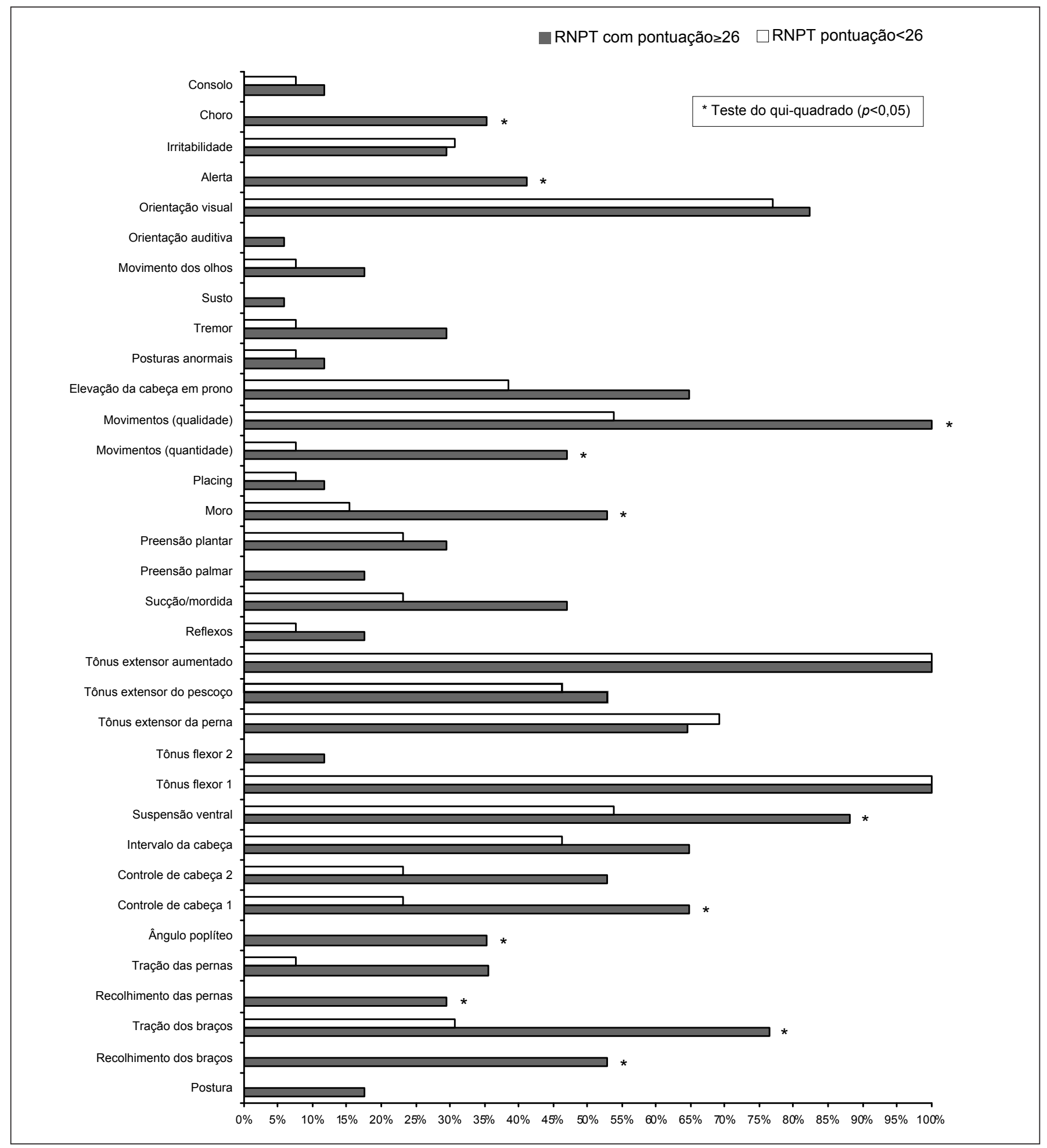

Gráfico 2 - Percentual de inadequação dos itens avaliados pelo método Dubowitz em recém-nascidos pré-termo.

de pontuações inferiores em $62 \%$ de RNPT em pesquisa na Nova Zelândia ${ }^{(10)}$ e, em $83 \%$, em Londres ${ }^{(18)}$. Em nosso estudo foi encontrado elevado percentual de RNPT com pontuação total inferior a 30,5 - o que sugere que tal ponto de corte, proposto inicialmente por Dubowitz ${ }^{(8)}$, provavelmente não é adequado ou que os RNPT com 37 semanas de idade pósconceptual podem realmente não apresentar comportamento semelhante aos RNT. 
Os próprios idealizadores do método, em estudo posterior, sugeriram a pontuação de referência 26 , que foi a pontuação média para RNPT ${ }^{(18)}$. Observaram também pontuações médias inferiores nas dimensões tônus e tipo de tônus, semelhantes às aqui observadas; porém, não foi encontrada a mesma diferença em relação aos reflexos e movimentos. Tal fato sugere que as diferenças no tônus possam ser características de RNPT, pois esses tendem a apresentar menor tônus flexor fisiológico, visto que, fora do ambiente uterino, não mais vivenciam a contenção uterina e a resistência ao movimento oferecida pelo líquido amniótico no último trimestre de gestação ${ }^{(26)}$.

$\mathrm{O}$ único estudo encontrado na literatura que utilizou a pontuação 26 de referência para RNPT foi o acima citado ${ }^{(18)}$. Porém, no presente estudo optou-se por adotar essa pontuação para possibilitar a melhor utilização dos dados referentes aos fatores de risco e análise mais acurada dos itens alterados nos RNPT, já que $90 \%$ deles não atingiram a pontuação 30,5 - o que prejudicaria a análise estatística.

Em nossa população, os RNPT que obtiveram pontuação total inferior a 26 apresentaram também pior desempenho nas dimensões tônus, tipo de tônus e movimentos; entretanto, o mesmo não ocorreu para os reflexos. As alterações nos reflexos provavelmente não apresentam relação direta com disfunções neurológicas futuras em RNPT, ao contrário dos movimentos ${ }^{(7,9)}$. As alterações de tônus e a assimetria de sua distribuição são enfatizadas na literatura como os principais indicativos de disfunções neurológicas futuras em $\mathrm{RN}^{(7,9,10,12,13,23-27)}$, diferentemente dos reflexos ${ }^{(10-12)}$. Em estudos nacionais e internacionais, as alterações do tônus são frequentemente associadas a lesões encefálicas diagnosticadas por exames de imagem, como a ultrassonografia (hemorragias peri e intraventriculares) ${ }^{(13,24)} \mathrm{e}$ a ressonância magnética (encefalopatia hipóxico-isquêmica) $^{(23)}$.

A combinação de alterações nas três dimensões (tônus, tipo de tônus e movimentos espontâneos) provavelmente possibilita a detecção de $\mathrm{RN}$ de risco para disfunções neurológicas. Essa hipótese foi apontada nos trabalhos de Woodward et $a^{(7)}$, Dubowitz et $a l^{(10)}$ e Molteno et a $l^{(7)}$. No estudo longitudinal de Woodward et al, os autores observaram associação significante dessas alterações com a presença de paralisia cerebral em 100 RNPT com um ano de idade. Dubowitz et al apontaram tais alterações como sinais neurológicos presentes em $82 \%$ dos RN com hemorragia intraventricular. O estudo de Molteno et al mostrou alterações no tônus em $70 \%$ dos $\mathrm{RN}$ com ressonância magnética de crânio anormal. O mesmo foi observado em $46 \%$ dos pacientes com o tipo do tônus alterado e em $56 \%$ daqueles com problemas relacionados aos movimentos espontâneos.
O único estudo que analisou as diferenças de pontuação entre RNT e RNPT de acordo com os itens individuais do método foi aquele adotado como referência para considerar a pontuação de 26 como ponto de corte de anormalidade ${ }^{(18)}$. Os RNPT obtiveram pontuação média inferior em 18 itens. Entretanto, todos os RN que participaram desse estudo clássico não apresentaram intercorrências clínicas no período neonatal, o que difere da presente pesquisa. Os demais trabalhos que compararam o desempenho dessas duas populações também referem diferenças na maioria dos aspectos ${ }^{(25,26,28)}$. Tais dados sugerem que há muitas diferenças entre os grupos, o que reforça que, provavelmente, o desempenho dos RNT não seria uma referência adequada para o desempenho de prematuros com idade corrigida próxima ao termo.

Atenção maior deve ser dada aos itens desviantes da avaliação dos RNPT, pois podem ser indicativos de lesão do sistema nervoso central. Woodward et al ${ }^{(7)}$ encontraram relação entre os itens qualidade dos movimentos, alerta e alterações do tônus em RNPT com lesões cerebrais detectadas por ressonância magnética. Outro estudo indicou associação entre alterações nos itens alerta, choro (irritabilidade) e tônus com a neuroimagem ${ }^{(29)}$. Nesse sentido, vários estudos mostram associação de desvios da avaliação neurológica com imagens de ultrassom sugestivas de lesão cerebral ${ }^{(10,13,25,30)}$, citando as modificações do ângulo poplíteo, quantidade dos movimentos, tônus, controle de cabeça, suspensão ventral, estado de alerta e quantidade de movimentos, entre outros.

Já nos estudos de acompanhamento do desenvolvimento dos RNPT $^{(11,12)}$, os itens referidos como preditivos de disfunções do desenvolvimento neurológico no primeiro ano de vida foram recolhimento e tração dos braços, controle de cabeça, suspensão ventral, quantidade dos movimentos e choro (irritabilidade).

Deve-se ressaltar que o presente estudo apresentou algumas limitações metodológicas: amostra de conveniência, com ampla variação do peso e idade gestacional ao nascimento, e cálculo da amostra baseado na prevalência de disfunções neurológicas em RNPT e RNT o que impossibilitou o emprego de análise estatística multivariada. Apesar dessas limitações, os RNPT aqui analisados na idade corrigida de 37 semanas apresentaram pior desempenho neurocomportamental quando comparados aos RNT; a presença de sepse neonatal se relacionou com os desvios da normalidade.

A realização de novas pesquisas, com ênfase em estudos de seguimento em longo prazo, será útil para relacionar os achados no período neonatal com alterações no desenvolvimento neurológico e assim, contribuirá para estabelecer ponto de corte específico para RNPT em nossa população. 


\section{Referências bibliográficas}

1. Ohgi S, Fukuda M, Akiyama T, Gima H. Effect of an early intervention programme on low birthweight infants with cerebral injuries. J Paediatr Child Health 2004;40:689-95.

2. Wilson-Costello D, Friedman $H$, Minich N, Fanaroff AA, Hack M. Improved survival rates with increased neurodevelopmental disability for extremely low birth weight infants in the 1990s. Pediatrics 2005;115:997-1003.

3. Sankar C, Mundkur N. Cerebral palsy-definition, classification, etiology and early diagnosis. Indian J Pediatr 2005;72:865-8.

4. Mello RR, Dutra MV, Silva KS, Lopes JM. The predictive value of neonatal neurological assessment and neonatal cranial ultrasonography with respect to the development of very low birth weight premature infants. Rev Saude Publica 1998;32:420-9.

5. Aparna S, Ravindra S. Predicting development delay in high risk infants: comparison between Dubowitz and Vojta Scale. J Assoc Physiotherapists India 2005;53:5-10.

6. Pedroso FS, Rotta NT. Neurological examination in the healthy term newborn. Arq Neuropsiquiatr 2003;61:165-9.

7. Woodward LJ, Mogridge N, Wells SW, Inder TE. Can neurobehavioral examination predict the presence of cerebral injury in the very low birth weight infant? J Dev Behav Pediatr 2004;25:326-34.

8. Dubowitz L, Mercuri E, Dubowitz V. An optimality score for the neurologic examination of the term newborn. J Pediatr 1998;133:406-16.

9. Dubowitz L, Ricci D, Mercuri E. The Dubowitz neurological examination of the full-term newborn. Ment Retard Dev Disabil Res Rev 2005;11:52-60.

10. Dubowitz LM, Levene MI, Morante A, Palmer P, Dubowitz V. Neurologic signs in neonatal intraventricular hemorrhage: a correlation with real-time ultrasound. J Pediatr 1981;99:127-33.

11. Dubowitz LM, Dubowitz V, Palmer PG, Miller G, Fawer CL, Levene MI. Correlation of neurologic assessment in the preterm newborn infant with outcome at 1 year. J Pediatr 1984;105:452-6.

12. Molteno C, Grosz P, Wallace P, Jones M. Neurological examination of the preterm and full-term at risk for developmental disabilities using the Dubowitz Neurological Assessment. Early Hum Dev 1995;41:167-76.

13. Sanchez-Stopiglia M, Moura-Ribeiro MV, Marba S. Neurological evaluation of neonates with intraventricular and periventricular hemorrhage. Arq Neuropsiquiatr 1999;57:366-70.

14. Hulley SB, Cummings SR, Browner WS, Grady D, Hearst N, Newman TB. Delineando a pesquisa clínica: uma abordagem epidemiológica. Porto Alegre: Artmed; 2003.

15. Battaglia FC, Lubchenco LO. A practical classification of newborn infants by weight and gestational age. J Pediatr 1967;71:159-63.

16. Capurro H, Konichezky S, Fonseca D, Caldeyro-Barcia RA simplified method for diagnosis of gestational age in the newborn infant. J Pediatr 1978;93:120-2.
17. Brazelton TB. Neonatal Behavioral Assessment Scale. Clin Dev Med 1973;50:53-9.

18. Mercuri E, Guzzetta A, Laroche S, Ricci D, VanHaastert I, Simpson A et al Neurological examination of preterm infants at term age: comparison with term infants. J Pediatr 2003;142:647-55.

19. O'Shea TM, Klinepeter KL, Meis PJ, Dillard RG. Intrauterine infection and the risk of cerebral palsy in very low-birthweight infants. Paediatr Perinat Epidemiol 1998;12:72-83.

20. O'Shea TM, Kothadia JM, Roberts DD, Dillard RG. Perinatal events and risk of intraparenchymal echodensity in very-low-birthweight neonates. Paediatr Perinat Epidemiol 1998;12:408-21.

21. Silveira RC, Procianoy RS. Ischemic brain damage in very low birth weight preterm newborn infants. J Pediatr (Rio J) 2005;81:S23-32.

22. Hieu NT, Gainsborough M, Simpson JA, Thuy NT, Hang NN, Taylor AM et al. Neurological status of low-risk Vietnamese newborns: a comparison with a British newborn cohort. J Health Popul Nutr 2006;24:57-63.

23. Mercuri E, Guzzetta A, Haataja L, Cowan F, Rutherford M, Counsell S et al. Neonatal neurological examination in infants with hypoxic ischaemic encephalopathy: correlation with MRI findings. Neuropediatrics 1999;30:83-9.

24. McGready R, Simpson J, Panyavudhikrai S, Loo S, Mercuri E, Haataja L et al. Neonatal neurological testing in resource-poor settings. Ann Trop Paediatr 2000;20:323-36.

25. Forslund M, Bjerre I. Neurological assessment of preterm infants at term conceptional age in comparison with normal full-term infants. Early Hum Dev 1983;8:195-208.

26. Mercuri E, Dubowitz L, Brown SP, Cowan F. Incidence of cranial ultrasound abnormalities in apparently well neonates on a postnatal ward: correlation with antenatal and perinatal factors and neurological status. Arch Dis Child Fetal Neonatal Ed 1998;79:F185-9.

27. Kurtzberg D, Vaughan HG Jr, Daum C, Grellong BA, Albin S, Rotkin L. Neurobehavioral performance of low-birthweight infants at 40 weeks conceptional age: comparison with normal fullterm infants. Dev Med Child Neurol 1979;21:590-607.

28. Palmer PG, Dubowtiz LM, Verghote M, Dubowitz V. Neurological and neurobehavioural differences between preterm infants at term and full-term newborn infants. Neuropediatrics 1982;13:183-9.

29. Dubowitz LM, Bydder GM, Mushin J. Developmental sequence of periventricular leukomalacia. Correlation of ultrasound, clinical, and nuclear magnetic resonance functions. Arch Dis Child 1985;60: 349-55.

30. Lazzara A, Ahmann P, Dykes F, Brann AW Jr, Schwartz J. Clinical predictability of intraventricular hemorrhage in preterm infants. Pediatrics 1980;65: $30-4$. 